\title{
Curriculum Management System Based on ADO.NET and B/S
}

\author{
Jun Yang \\ School of Education and Sports, Bohai University, Jinzhou, 121013, China \\ yj690213@126.com
}

Keywords: ADO.NET; B/S system architecture; curriculum management system; overall function

\begin{abstract}
This paper based on ADO.NET and B/S system architecture elaborates the design process of the curriculum management system and it also analyzes the superiority of .NET and B/S in practical application. First of all, this thesis states necessity of curriculum management system under the credit system. Then it designs the overall function of the system, mainly including students model, teachers model and administrators model. Again B/S structure including its basic ideas and the specific function of three levels is introduced in it. Eventually it introduces ADO.NET technology. The curriculum management system can not only help teachers to manage students better, but also can fully mobilize the enthusiasm of students learning to make students realize their self-management, provide guarantees, premises as well as theoretical basis for successful curriculum revolution.
\end{abstract}

\section{Introduction}

With the accelerating pace of information technology, modern information technology represented by network, digitization and intelligentialize is not only changing the way of people's work, study and life, but also has brought new development to education [1]. University education reform is deepening, credit teaching model that replace traditional teaching model gradually is implemented in various colleges and universities. Credit with students elective at the core and teachers' instruction for assistance, as an educational model, is a comprehensive teaching management system that through grade point and credits to measure the quality and quantity of students' learning. Such system that elective course replaces scheduling allows students arrange their learning plan according to their abilities and interests, or even change major at any time, and it not mandatory require students to go to class according to the school program. From the teachers, teachers can enhance the sense of competition which will help improve teaching effectiveness. Students choose their teachers according to their preferences that can urge teachers to prepare more classroom data. Schools can judge a teacher by the number of students; from the students, they can really choose their favourite courses to stimulate their interests in learning. This model not only enhances students' interest in learning the professional knowledge, but also to provide a guarantee system for training high-quality professionals in a field [2]. This model puts forward higher requirements for school curriculum management. Therefore, curriculum management system is designed to provide guarantee for curriculum management in this model with information technology as the basis.

\section{Overall Function Design of System}

The general function of system is designed as shown in the Fig. 1. According to different landing role, the system mainly divides users into teacher users, student users and administrators. These users manage the system separately from different aspects of the whole system in order to make the function module structures more clearly. There are various demands for different users, so the research designed the system because of different users' permissions.

(1) Teachers model: teachers land the system and then they mainly carry on curriculum management. The role function is mainly divided into five parts. Firstly, teachers could request courses according to what they have taught. After the successful application, students choose the course what they want to learn through this system, including the selection of teachers, the choice of course place and time; secondly, they could manage students' achievement, including usual grades, 
the mid-term grades, final grade and so on. Scores are given according to the usual performance and attendance of students, and these grades were released in a timely way to make reference for the students. In view of the curriculum reform, the final result mainly decided by the scores at ordinary times and it's computing method is: $S=60 \% \times S_{1}+40 \% \times S_{2} . S_{1}$ stands for usual grades and $S_{2}$ stands for final grades in the formula; thirdly, they could upload their own teaching video recordings or other valuable videos downloaded from the Internet to the server, also they can upload questions what were summarized according to their own experience in teaching process about making the students understand themselves and remember the knowledge to let students learn after class and improve students' interest in learning; fourthly, teachers can communicate with students online to help answering questions students don't know; finally, they could manage their own information, such as uploading photos, updating their personal data. They can record and view all operations on the Internet as well, such as uploading video, releasing grades and exchanging information, etc.

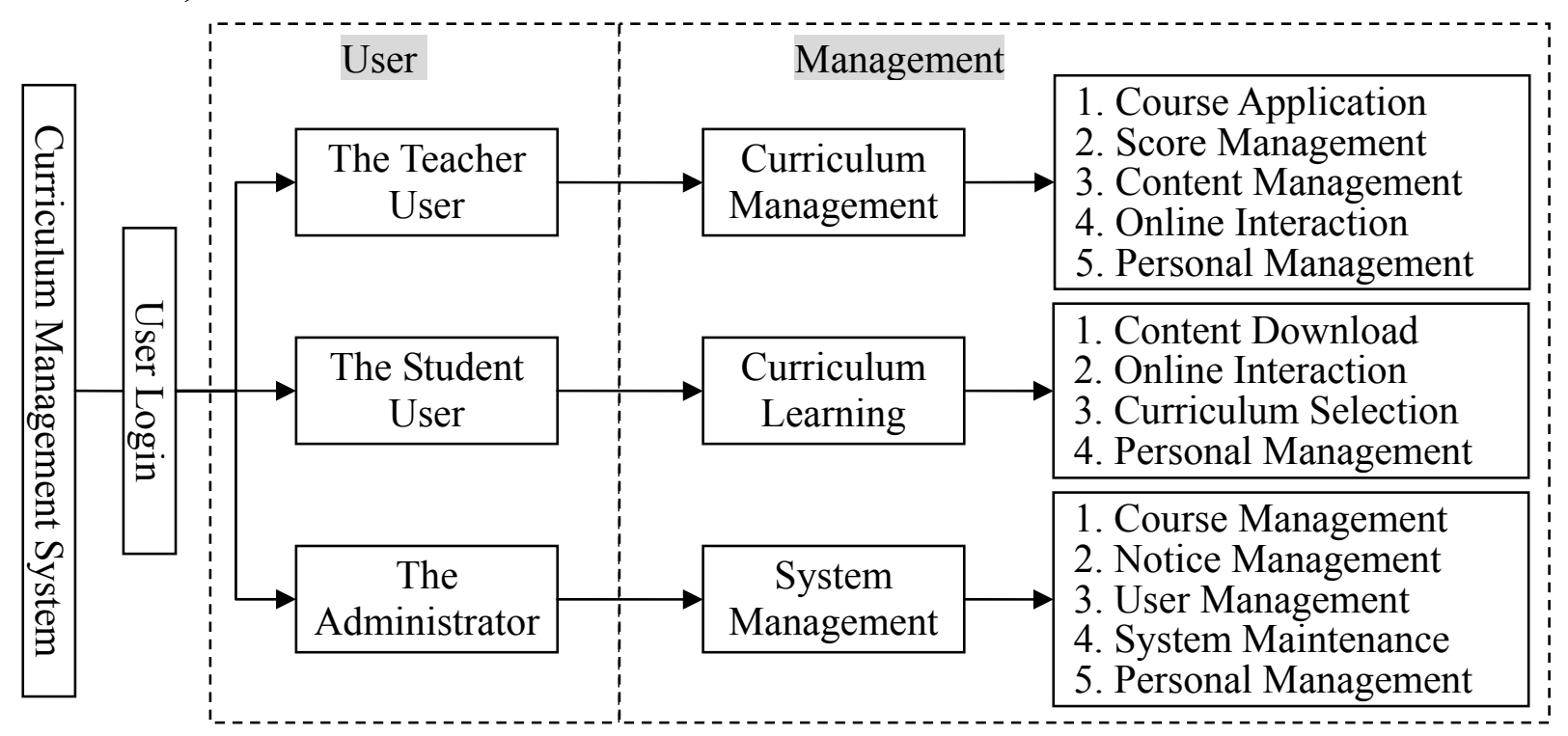

Fig. 1. Functional block diagram of system

(2) Students model: students land the system and then they mainly study curriculum. The role function is mainly divided into four parts. Firstly, download learning video or data according to their own interest or doubt; secondly, communicate with the online teacher timely aiming at the their own problems or give teachers a message waiting for reply to achieve further understanding; thirdly, students can look over the arrangement information of curriculum and teachers, also they can use the query function looking at the course teaching plan and teachers' information to choose their favourite courses and understand related teaching information of courses; fourthly, they could manage their own information, such as uploading photos, update their personal data. They also can record and view all operations on the Internet, such as data download, course selection and communication of information, etc.

(3) Administrators model: administrators land the system and then they mainly carry on managing the system. The role function is mainly divided into five parts. Firstly, they can manage teachers' apply of courses arranging, including time, location, and the number of student; secondly, releasing information is the first step of announcement management, releasing information function can satisfy the multiple levels of the functional requirements of users. Administrator should be able to modify and delete announcement at any time in order to ensure the public announcement of the dynamic characteristics of information management; thirdly, they can supervise and control the users (teachers and students). They can query, modify, and delete operations for users; fourthly, they could access IP address, manage background operation log, etc; finally, they could manage their personal information, such as uploading photos, updating their personal data. And they can also record and look over all operations on the Internet, such as releasing announcement, managing course and user, etc. 


\section{System Structure Design}

At present, the development model for software system mainly includes B/S (Browser/Server) model and C/S (Client/Server) model. Due to develop the curriculum management system, specific operators can be either a system administrator or outside visitors, therefore, $\mathrm{B} / \mathrm{S}$ system development model can meet the functional requirements of the system for users. B/S three-tier structure includes presentation layer, business layer and data layer from top to bottom. System takes thought of "divide-and-conquer", the problems are solved separately by type, the changes of presentation layer will not affect code written of another two layers, and the changes of data layer will not affect the design of presentation layer. Such program structure is easy to control, extend and allocate resources in order to achieve "high cohesion, low coupling", and the B/S is a very popular program architecture [3].

Presentation layer: it, located in the outermost layer, mainly refers to the operation interface that interacts with users. This layer provides a variety of operation interfaces, such as the login interface, the program application interface, the real-time interaction interface and personal information interface and so on. Users issue requests to the Web server on the network through the browser, and Web server submits requests of the browser to the data operation interface in business layer, then returning information required by users to the browser.

Business layer: it is mainly responsible for the operation of the data layer, which is located in the middle of the presentation layer and data layer, and it plays a connecting role and is the core sector to achieve the business logic and data manipulation. Its mission is to accept the user's requests, first implementing scalable applications and linking with database, and putting forward data processing applications to database server by way of the SQL, then database server will submit the results of the data processing to the Web server, and final the results will be back to the client by the Web server.

Data layer: this layer is responsible for database access operations, including increase, delete, modify, query and other operations, and accepting requests from the server, specifically to provide data services for business layer or presentation layer. This layer stores all the information tables, including user information table, curriculum table and so on [4]. Three-tier architecture diagram of system is shown in Fig. 2.

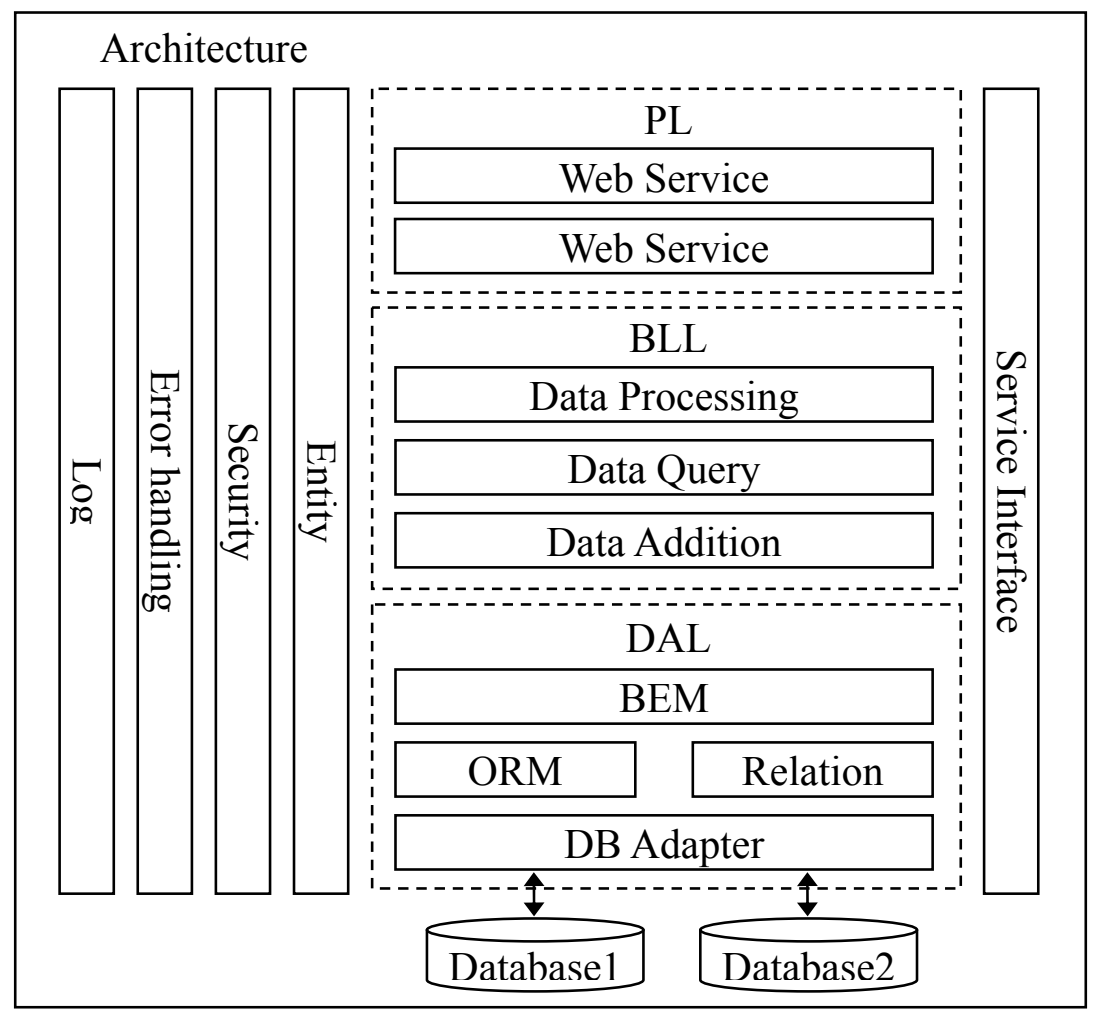

Fig. 2. Architecture diagram of system 


\section{Key Technology}

The name of ADO.NET is originated from ADO (ActiveX Data Objects), and it is a COM component library for accessing data in previous Microsoft technologies [5]. ADO.NET is a redesigned, new generation of data access object for the shortcomings of ADO with some increase compared with the original ADO structure, which supports the exchange of the underlying data type, its object model is different with ADO of early database. Using ADO.NET technology not only can access the data in the database, but also access to the hierarchical XML data. ADO.NET provides two data access models for application: connected mode and disconnected mode. Compared with the traditional model, disconnected mode reduces the time for connecting and disconnecting a database, so its work efficiency will be improve to provide the reliability and stability of the application. For distributed applications, ADO.NET also can significantly improve the performance of the program. ADO.NET class consists of two parts: data provident (sometimes also called hosting provident) and dataset. Its object model is shown in Fig. 3.

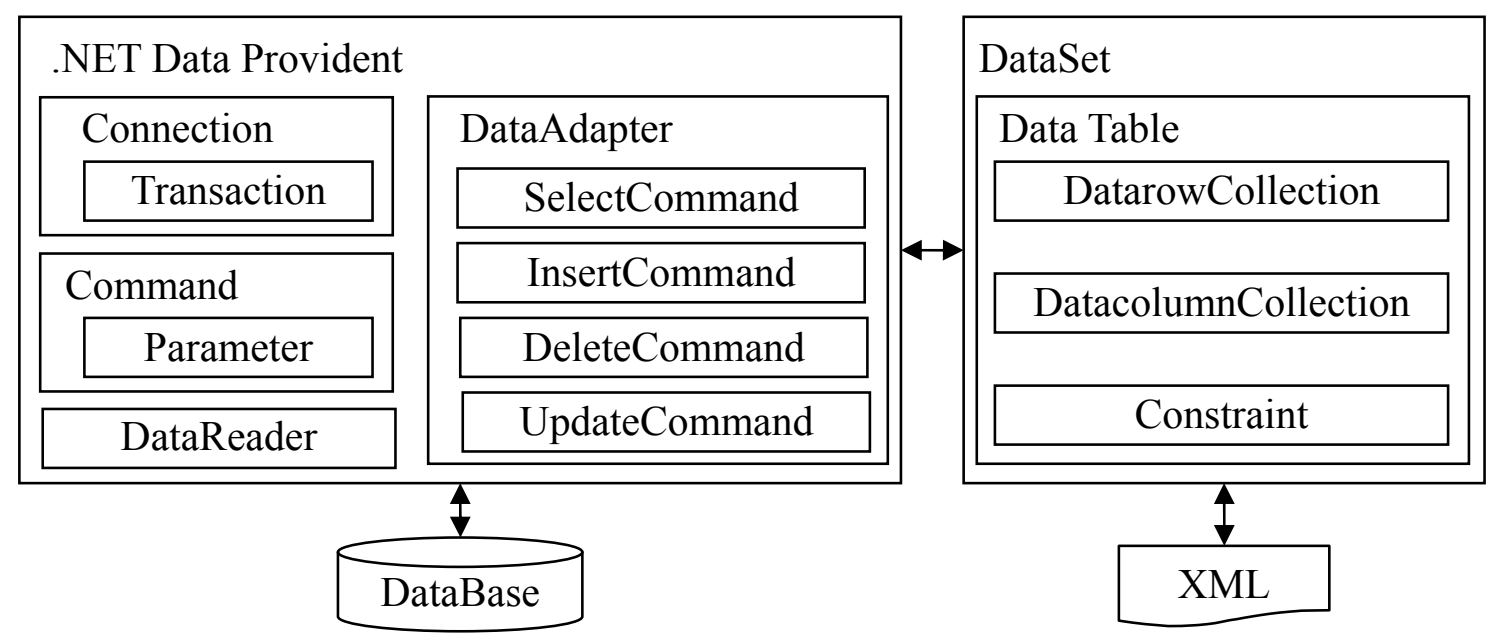

Fig. 3. The object model of ADO.NET

(1) Connection object: it is used to establish a connection between the application and the database, but you must provide the name of the database, the computer source, the type of data provider, user name and password. .NET framework mainly consists of two Connection objects, respectively SQL Connection and ADO. Connection. First, using SQL Connection to establish object, and then linking the data source supported ADO Managed Provider.

(2) Command object: it is used to set the SQL commands, transfer the system parameters and get the data results and use commands to edit, delete, retrieve and insert data to the database. It can call stored procedures in a database. This object is built on the Connection object and is linked to data source also through the Command object.

(3) DataSet object: the object, the core of ADO.NET, can be seen as a cache. It is a memory-resident representation of data, it will provide a consistent relation programming model regardless of the data source. It can be used to many different data sources, such as XML data, or data of local applications. DataSet means the entire data set, including inter-related tables, constraints, and table relationships.

(4) DataAdapter object: it is the bridge for connecting DataSet object and data source, ADO.NET creates, initializes data table by DataAdapter object, so it is integrated with DataSet object to store data in memory. It also allows you to return the changing data in DataSet to the database [6]. Similarly, the underlying data can also be added, deleted, or modified. This object is built on Command object and it provides a number of functions that used with the DataSet.

\section{Conclusion}

Information technology is needed to manage curriculums under the reform in the information age, and the theory of curriculum management system in this paper put forward constructive ideas and 
processes, which provides a theoretical basis for the actual development of the curriculum management system [7]. This paper designs curriculum management system based on .NET and $\mathrm{B} / \mathrm{S}$ system architecture, and uses a set of core class libraries to provide service for the applications, which can reduce a lot of basic work to make the software structure more clear, so designers can clear division of labor. ADO.NET, developed over ADO, is a new model for data manipulation specifically for data access operations on the .NET platform based on .NET Framewok. .NET has many advantages, such as the fast running speed, the flexible encoding model, and a higher efficiency in the developing small and medium projects. And it occupies less resource due to use non-connected model. B/S structure is a network structure model after Web rise, and this model unifies the client, concentrates the core part of the system function realization on server, and so simplifies the development, maintenance and use of system. With the continuous development of the theory of educational technology and network technology, the research and exploration for teaching platform will continue in-depth, so the platform will have broad application prospect in modern teaching. The development and design of the system can make management work become relatively easy, and data are not easy to lose and can be stored longer, which will bring great convenience to the work of teachers and the learning of students [8-10].

\section{Acknowledgement}

This work is supported by A-class teaching reform project of Liaoning Education Department in 2014 (UPRP20140249), The key issue of Liaoning economic and social development in 2015 (20151slktzimzs-02), A-class teaching reform project of Bohai University in 2013 (JG13YB004), "Twelfth Five Year" planning project of Liaoning education and science in 2013 (JG13CB030).

\section{References}

[1] H. B. Sun. The Design and Implementation of Excellent Course Management System. Master's Degree of Shandong Normal University, 2014.

[2] D. Y. Shi, Z. S. Jia. Application of J2EE Lightweight Framework in College Curriculum Management System. Computer and Modernization, 25(8), pp. 152-156, 2009.

[3] D. Kang. Design and implementation of course management system for project-based teaching. Master's Degree of Sun Yat-sen University, 2013.

[4] P. F. Li. Design And Implementation of Management System for Comprehensive Course in University Based on .Net. Master's Degree of Yanshan University, 2012. http://baike.baidu.com/link?url=GQ0b8OcCNBVzmorfIwKTw5QVa4wFkEle41Wf9MubG6kb s0UVcuTzzebaI0c2tf0Ez615FGnwVuAb7Wa30IB8dq, 2015-4-5.

[6] W. Jiang, Q. G. Lai, L. Qin, X. B. Zhang. Research and Application of Data Access Technology Based on ADO.NET. Microcomputer Information, 27(30), pp. 141-143, 2010.

[7] H. H. Yin. The Study and Realization of University Curriculum Management System. Master's Degree of Jilin University, 2009.

[8] Baidu Baike. B/S. http://baike.baidu.com/link?url=Jb8wk_1codl_GINSeO6F-9g6jQ8wK, 2015-4-6.

[9] Z. G. Bi, J. P. Xu, X. G. Huo. Research on the Design and Application of B/S Based Teachers' Course-Management System. Software Guide, 10(3), pp. 129-141, 2011.

[10] X. L. Yang. Based on B/S Model ASP.NET Technology in the Student's Dormitory Management System Application. Computer Programming Skills \& Maintenance, 18(2), pp. 70-71, 2011. 